\title{
Metodología y tecnologia cualitativas: actualización de un debate, desde la mirada más atenta en la obra de Barney G. Glaser ${ }^{1}$
}

\author{
Miguel S. VALleS \\ Departamento Sociología IV \\ Universidad Complutense \\ mvalles@cps.ucm.es
}

Recepción: diciembre 2004

Aceptación: marzo 2005

Para Aniceto Martínez Poza, In Memoriam.

\section{INTRODUCCIÓN: CONTEXTO EXISTENCIAL EN LA INVESTIGACIÓN DE UNA RELACIÓN POLÉMICA}

Sigue la disputa sobre si son mayores los beneficios o los perjuicios de las nuevas asistencias de la tecnología en el viejo oficio de la investigación social. El recuerdo, homenaje, de la contribución pionera de Renata Tesch (1990) da cada vez más perspectiva a una cuestión formulada de muy diversas formas en el terreno particular de la investigación social cualitativa ${ }^{2}$. Hay en ello no sólo interrogantes explícitos o manifiestos, sino latencias que sitúan el debate en un plano también epistemológico. Unos y otras relacionados a modo de tupida red de conexiones que conviene destejer, para dar cuenta de la malla conceptual ${ }^{3}$ que

' Este artículo es una versión reelaborada de la ponencia presentada en el VIII Congreso Es-

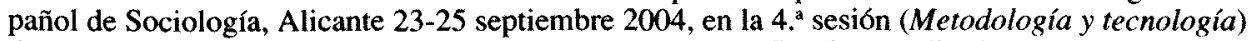
del grupo de trabajo 01-Metodología. Agradezco a Ercilia García y Jordi López su apoyo bibliográfico.

${ }^{2}$ Por ejemplo, a la pregunta primera, más general y directa, sobre el porqué del uso de ordenadores en la investigación cualitativa (Fielding \& Lee 1998: cap. 3), los mismos autores añaden otras, más específicas, en 2002. A saber: ¿se trata (el software que asiste en el análisis cualitativo) de una fuente técnica o de un enfoque analítico?; ¿cuáles son las nuevas pautas en su adopción y uso?

${ }^{3}$ No es otro el planteamiento del propio Glaser (2001), cuando contrapone la conceptualización a la mera descripción; la generación de conceptos, enraizados en los materiales del trabajo investigador, a la mera aplicación de conceptos concebidos por otros. 
permita entender e interpretar la experiencia vivida ${ }^{4}$ en este campo. La perspectiva histórica no puede faltar, tampoco la biográfica.

Mi particular aproximación a este terreno ha ido dejando un rastro documental, más exteriorizado, (Valles 1997: cap. 9; 2000; 2001); y otro, menos visible, en el archivo más personal de las headnotes (Ottenberg, 1990). Este último, un recurso investigador muy útil y utilizado, el de la instrospección. La vieja técnica del autorretrato, reivindicada en un momento de la historia de las ciencias sociales en el que las observaciones exógenas o endógenas de lo social no se entienden sin un mínimo de autoobservación del caso individual más próximo. A este respecto, advierto al lector, desde el principio, que quien escribe lo hace condicionado por su particular experiencia biográfica en relación con la llegada de las nuevas tecnologías (hardware y software) al contexto de la investigación sociológica española.

Permítaseme esta sucesión de instantáneas sociológicas, que quieren recordar sin conseguir emular las así denominadas por del emérito profesor hoy, José Castillo, en su conferencia de clausura del VI Congreso de la FES en 1998 , en A Coruña. A finales de los 80 llegaba el primer ordenador personal (un IBM XT) al despacho privado de sociología de Amando de Miguel, en Serrano, 19; y en esas mismas fechas lo hacía en el Departamento de Sociología IV (Metodología de la Investigación) de la UCM, donde el jovencísimo profesor Modesto Escobar aconsejaba a los becarios José Ignacio Cano Gestoso, José Melquíades Ruíz Olano y Miguel S. Valles que había que «dejarle tiempo» al ordenador para que ejecutase la orden de análisis factorial (factor variables $=..)^{5}$. Enseguida, a principios de los 90, llegarían los AT a ese Departamento, junto con la posibilidad de una oferta para los profesores que hizo realidad nuestro primer sueño del equipo (ordenador, más impresora) en casa ${ }^{6}$. Las peticiones, tasadas, de tabulaciones de estadísticas a las administraciones públicas iban a acabar. En el verano de 1992, tras los cursos en la Universidad de Essex encargué el TEXTPACK (mi primer software para el análisis de contenido clásico de material textual) ${ }^{7}$. Si bien, entre el acopio de

${ }^{4}$ Sobre el concepto experiencia vivida y su relación con la génesis de la investigación cualitativa véase Hamilton (1994), reseñado en Valles (1997: 22-24).

${ }_{5}$ Por esas fechas, (1987) Julio Cabrera publicaba un escrito pionero, que sirve de documento histórico de una época en la que algunos investigadores, fuera y dentro de España, producían software para autoconsumo en su práctica investigadora orientada hacia el estudio de casos.

${ }^{6}$ Cuando escribía la primera versión de estas líneas (junio 2004) se acababa de hacer público el convenio de la UCM con una empresa informática, para ofrecer ordenadores portátiles con tecnología sin cables a precios más económicos a alumnos y personal.

${ }_{7}$ El programa TEXTPACK era el utilizado en el curso Textual Analysis: the language of politics, impartido en la Escuela de Verano de la Universidad de Essex por Ekkehard Mochmann (Universidad de Cologne), quien ya había publicado en la RIS, en el monográfico coordinado por J.Javier Sánchez Carrión años antes (Mochmann 1985). Hoy se presenta, en la red, como «TEXTPACK: Un sistema para el análisis de contenido cuantitativo asistido por ordenador»; y se ofrece en inglés y en español en la dirección: http://www.gesis.org/en/software/textpack/. 
libros adquiridos en las librerías de Oxford, Cambridge y Londres estaban los de Tesch (1990) y Fielding \& Lee (1991). Poco después, 1993, Benjamín González me pondría al corriente de las primeras versiones de NUDIST ${ }^{8}$, cuando aún el sistema operativo MS-DOS primaba.

Volviendo al hardware, recuerdo la evolución rápida de los procesadores $286,386,486$ en esos años 90 . Todos los despachos del Departamento mencionado acabarían teniendo un ordenador e incluso se produciría la renovación de los modelos más antiguos. En el ámbito doméstico y, precisamente, para poder utilizar el nuevo software que iba apareciendo, ya en la plataforma Windows, el salto a un pentium $200 \mathrm{MMX}$, a comienzos de 1998 , supuso la conservación como pieza de museo del AT. Los 40 megas de disco duro, se multiplicaban por 100 , y se hablaba en gigas $(\mathrm{Gb})$. De la pequeña pantalla en blanco y negro al monitor a color, con más pulgadas y digital. Entre otras muchas innovaciones tecnológicas, la que merece destacarse aquí es el CDRom. Thomas Muhr, autor del programa ATLAS/ti, se había anticipado (en 1997) con su versión 4.1 bajo Windows, en un disco compacto, al lanzamiento en este mismo formato de NUD*DIST NVivo (en marzo de 1999) por los Richards. La novedad del soporte CD-Rom, frente a los usuales discos de $3.5^{\prime \prime}$, de 1,44 Mb de memoria, traía consigo otra de enorme interés para el autoaprendizaje. La capacidad de almacenamiento en un solo disco compacto, multiplicada por más de 400 , permitía incluir, junto con el software del programa, grabaciones audiovisuales sobre el funcionamiento de algunas rutinas e incluso tutorials (Valles 2000: 576). Por último, las limitaciones de memoria RAM y de velocidad del procesador en el uso del software mencionado, para proyectos de mayor envergadura, se han visto resueltas con el hardware más actual.

Este pequeño carrusel de instantáneas, sin pretensión de exhaustividad, sirve además para subrayar la rapidez de los cambios habidos en este terreno. La actualización de este campo, abierto, de investigación y reflexión metodológica se hace perentoria y se atisba interminable. El ritmo de los upgrades del software y del hardware o la aparición de nuevos productos ha dejado obsoletos, en poco tiempo, diagnósticos laboriosos como los de Weitzman y Miles (1995) ${ }^{9} \mathrm{o}$ el de Barry (1998). Si bien, la cuestión metodológica de fondo, sobre la que se centra el foco de nuestra atención aquí, sigue vigente.

${ }^{8}$ Nonnumerical Unstructured Data Indexing Searching Theorizing. En el manual de la versión 3.0 para $\mathrm{PC}$ con sistema Windows, con fecha mayo de 1994, se definía como «software diseñado para ayudar a los investigadores en el manejo de Datos No Numéricos, Desestructurados, mediante procesos de Indexado, Búsqueda y Teorización». Esta era la versión PC 386 anunciada en Fielding \& Lee (1991: 198) para finales de 1991, en un libro que informaba de la versión 2.1, sólo para Macintosh u ordenadores grandes (mainframe version).

${ }_{9}$ Por ejemplo, la valoración que hicieran Weitzman \& Miles (1995: 133-136) del programa MAX ya no se sostiene en el caso de winMAX y, menos aún, de MAXqda (Valles, 2002). Tres versiones de un producto que sigue desarrollándose (Maxqda Dictio). Otro ejemplo está en los sucesivos modelos lanzados por la firma QSR: NU*DIST, NVivo, XSight. 


\section{ANTECEDENTES, RELEVANCIA Y ACTUALIDAD DEL DEBATE: UNA SELECCIÓN DE BALANCES Y PRONÓSTICOS EN BUSCA DEL POSO CONCEPTUAL}

La investigación de Fielding y Lee (1998), a pesar de los años transcurridos, constituye aún un extraordinario minarete desde el que otear el paisaje aquí enfocado, sin perder la perspectiva temporal. El último capítulo del libro citado se planteó primero como un ejercicio de anticipación, pero acabó escribiéndose en términos de «evolución». Allí se encuentra una aportación conceptual que sirve para ir esbozando el debate. A saber: «los importantes desarrollos tecnológicos que experimenta actualmente la investigación cualitativa no llevan al análisis cualitativo a destinos nuevos sino que están causando la vuelta a viejos ${ }^{10}$ debates» (Fielding y Lee 1998: 181). No es inmediata la comprensión de este aserto. Para tratar de abrir buena parte de lo que encierran estas palabras hay que perfilar algo más el mensaje de estos autores. Se trata de un pronóstico con claros y oscuros, acerca de los efectos de lo tecnológico en lo metodológico. Vislumbraban entonces un amplio margen de «experimentación» " e «innovación», teñido de «confusión» pero sin hipotecar un final feliz. En otras palabras, un pronóstico optimista de cambio no revolucionario que apuntaba un cierto potencial transformador de las nuevas tecnologías y se saldaba en un balance de innovación metodológica. Ya en esas fechas (1997-98), las crecientes cifras de ventas de programas especializados en el análisis de material cualitativo no son el único indicador sobre el que apoyan sus previsiones. Hay un interés por los nuevos productos que se traduce en estudios de mercado, aparición en revistas especializadas, penetración en nuevas disciplinas y en la programación de los cursos de métodos de nivel de licenciatura.

En los años siguientes se ha ido apreciando mejor que la llegada de Windows fuese un estímulo para mejorar algunos programas (otros han desaparecido ${ }^{12}$ ); y que la lista de deseos de los usuarios de las primeras versiones cada vez sea más pequeña (Valles 2001). Ciertamente, hay pendientes cuestiones como la trascripción, si bien ya han ido desarrollándose algunas soluciones que suponen un

${ }^{10}$ Este es mi caso. El enfoque en la relación investigación cualitativa y recursos tecnológicos me ha llevado al redescubrimiento de la grounded theory, de Glaser, de sus maestros, de las raíces sociológicas y metodológicas previas.

${ }^{11}$ El período de experimentación se fecha a principios de los años 80. Los «investigadores cualitativistas exploraban el uso de las bases de datos, los programas de análisis de contenido y los procesadores de texto». Yo añadiría que es el inicio de un largo período de transición: primero versiones rudimentarias, luego desarrollo de versiones PC. Hasta llegar al surgimiento de una «comunidad internacional» de fabricantes y usuarios y otros «interesados en los aspectos técnicos y metodológicos de CAQDAS», con «conferencias regulares, grupos de usuarios, boletines electrónicos y una creciente literatura sobre el tema» (Fielding y Lee, 1998: cap. 1).

12 A fecha de 2004 cabe añadir que otros programas no han llegado a desaparecer pero su obsolescencia se divulga. Es el caso de Ethnograph, "la grande dame del análisis cualitativo asistido por ordenador no se ha actualizado desde 1998, y ha quedado obsoleto», según se lee en la página del Departamento de Ciencias Sociales de la Universidad de Loughborough (http://www.lboro.ac.uk/research/mmethods/research/software/caqdas.html). 
avance respecto al software de reconocimiento de voz. El análisis directo del discurso hablado (gracias a la grabación digital y sus posibilidades de codificación), pudiera traer consigo modos de análisis distintos a los empleados tradicionalmente con el discurso escrito. Sin duda, está por investigarse si la irrupción de los ordenadores en la especie humana incrementará en ésta no sólo el uso de su capacidad visual sino también la auditiva, frente a la meramente lectora.

Pero el planteamiento más elaborado de los promotores del CAQDAS Networking Project ${ }^{13}$ no señala sólo a las nuevas tecnologías como el motor del cambio en el análisis cualitativo. «La tecnología transforma, pero no es el único agente de transformación», sentencian como colofón de su obra. El otro gran agente, anterior al tecnológico, lo constituyen las diferentes escuelas y enfoques en la investigación cualitativa. Si bien, no comparten la visión dicotómica de Coffey y otros (1996), al emparejar codificación o indexado con teoría fundamentada e hiperenlaces o referencias cruzadas con análisis postmodernista. El recurso al punto de vista de Kelle (1997) resulta conciliador y elocuente de un planteamiento atento a una práctica investigadora más mixta que la predicada en ocasiones desde la disputa metodológica. El uso simultáneo de técnicas de indexado y de referencias cruzadas, desde los tiempos más remotos de las exégesis bíblicas (hasta los más actuales del «uso creativo» de estrategias analíticas con tradición ${ }^{14}$ ) así lo atestigua; y desdice a los que sostienen que las diferentes perspectivas analíticas estén inscritas en la técnica o en la tecnología.

La investigación señera de Fielding y Lee (1998) contiene otras muchas aportaciones a la relación enfocada aquí, entre metodología y tecnología cualitativa, algunas de la cuales retomaremos en los apartados siguientes. Ahora conviene señalar que a ella le han seguido trabajos más recientes (Fielding 2000; Gibbs, Friese y Mangabeira 2002; entre otros), en los que se sigue practicando el ejercicio intelectual de pronosticar el futuro a partir de las pautas emergentes detectadas en el presente. Interesa anotar sobre todo su aportación conceptual. Por ejemplo, Fielding pronostica una creciente aceptación del software en el cvitae metodológico y la mayor accesibilidad a las publicaciones de una nueva generación de académicos con una noción más clara de las posibilidades y los límites de dicho software. Pero la tendencia de mayor alcance, confirmada en los resultados más recientes del CAQDAS Networking Project, reside en el aumento de los usuarios no académicos con escasa formación en ciencia social. Lo que está planteando una autonomía creciente de la investigación social hecha desde disciplinas diferentes (por personal sanitario, por ejemplo). En

${ }^{13}$ Sobre esta investigación cualitativa continua, iniciada a principios de los 90 , acerca de las experiencias de los usuarios de software para el análisis de datos cualitativos en el Reino Unido véase: Fielding \& Lee 1998: 2-5; 2002; y la dirección http://caqdas.soc.surrey.ac.uk/. CAQDAS responde a la expresión Computer Assisted Qualitative Data Analysis Software; i.e. software para el análisis de datos cualitativos asistido por ordenador.

${ }_{14}$ Se refieren Fielding y Lee (1998: 189) al interaccionismo simbólico, el análisis de la conversación y la teoría fundamentada. Sobre la disputada cuestión de las tradiciones en la investigación cualitativa véase Hamilton (1994) y Crewell (1998). 
suma, la distinción de usuarios, la cuestión generacional y las expectativas imaginadas desde la confusión entre herramienta y técnica son algunas de las claves conceptuales.

Por su parte, Gibbs, Friese y Mangabeira (2002: 8-10) trazan un panorama futuro definido por la convergencia digital, las nuevas cuestiones éticas, los «estándares digitales emergentes» (tipo XML), el reto de la integración de la enseñanza del software y su reconocimiento académico, la expansión del soporte a otros enfoques analíticos. No obstante, su balance está atravesado en buena medida por el escepticismo que ha suscitado y sigue suscitando la adopción de las nuevas herramientas en el taller artesanal de la indagación cualitativa. La prueba de la generalización de las nuevas tecnologías en la investigación cualitativa se condiciona al «uso de nuevas formas de datos y tipos de análisis que no se hubieran pensado tan siquiera en el pasado de lápiz y papel». Reconocen que aún es discutible si se ha llegado o no a dicha situación. Pero, en su opinión, el monográfico que presentan aporta prueba suficiente en esa dirección. En todo caso, su concepto de impacto dual, de la nueva tecnología en la investigación cualitativa, deja más claro (que en los escritos de los autores anteriores) que no es sólo en el análisis final donde se producen los efectos, sino en el registro y recogida de la información también. Este planteamiento coincide con el dado aquí, por nosotros, al formular la cuestión de fondo como una relación entre lo tecnológico y lo metodológico. Enseguida se verá que algunas posturas, abiertamente contrarias a las nuevas tendencias, cabe calificarlas de tecnófobas y no sólo en sentido figurado, como la que se toma de epicentro en este escrito, la que representa Glaser.

Finalmente, anoto brevemente una aportación conceptual propia. Trato de expresar, con la economía del lenguaje conceptual, la experiencia vivida en los últimos años a propósito de la investigación cualitativa y la irrupción de la microinformática. Un modo sintético de nombrar lo ocurrido estaría en las expresiones contrapuestas: tradición metodológica y transición tecnológica. La primera alude a un cierto clasicismo o permanencia metodológica. La asociación principal (que no exclusiva) la establezco con la propuesta que hicieran Glaser y Strauss (1967). Todo un esfuerzo de sistematización del análisis cualitativo, pero no sólo; pues se trata de una perspectiva teórica y metodológica abierta también a los datos cuantitativos (Glaser, 1993). En todo caso, cabe recordar que surge en los años 60, en la sociología norteamericana, con la pretensión de superar la concepción de la teoría y la metodología transmitida por Merton y Lazarsfeld, tratando de integrar los modos de hacer de la Escuela de Chicago ${ }^{15}$. Pero es el segundo concepto, el de transición tecnológica, el que interesa destacar más ahora. Debido a mi formación y especialización inicial en el campo de la sociología de la población, el modelo teórico de la transición demográfica ha actuado de resorte a la hora de elegir un código como el propuesto para hacer ba-

${ }^{15}$ En otro lugar, he trazado algunas pinceladas sobre la recepción de la grounded theory y los programas de análisis cualitativo en España (Valles 2002). 
lance y pronóstico de la relación metodología y tecnología cualitativas, desde el contexto español de hoy. Si, en lo demográfico, el modelo teórico mencionado ha servido para conocer la experiencia histórica de diversos países caracterizados por pasar de un régimen antiguo a otro moderno; en lo tecnológico, diríamos que alude al paso de un uso de tecnología rudimentaria (con predominio de los modos manuales) a otros usos provistos de recursos tecnológicos más o menos avanzados o complejos (incluidos los sistemas de enlaces y redes a gran escala). En estos últimos estadios destacaría el empleo de diversas modalidades de hardware (no sólo el ordenador) y de software ${ }^{16}$. El concepto matriz de transición tiene la ventaja de subrayar el carácter procesual de lo que se estudia. Además, se espera que, de manera similar a lo ocurrido en la sociología de la población, la expresión propuesta haga funciones de paradigma e independientemente de sus anomalías promueva la investigación. ¿En qué fase de la transición tecnológica nos encontramos?; ¿cuáles están siendo las pautas de adopción y uso de las nuevas tecnologías en la investigación social cualitativa en España?; ¿qué procesos de recepción y adaptación tienen lugar? Estos son algunos de los interrogantes que cabe plantearse y sobre los que habrá que seguir investigando.

\section{DE LAS VENTAJAS E INCONVENIENTES A LAS TIPOLOGÍAS DE PROCESOS, CASOS Y POSTURAS}

Pensar en términos de suma y resta, beneficios y perjuicios, parece consustancial a la actividad humana; a la experiencia vivida; a nuestra memoria personal y colectiva. Todavía recuerdo la instalación de la primera impresora, ayudado por un amigo ingeniero de telecomunicaciones. Probando la rapidez y calidad de impresión surgió el comentario sobre el coste en ruido que las opciones distintas a la de «borrador» implicaban, de lo que nada podía leerse en el manual de instrucciones. Enseguida aparecerían en el mercado modelos más silenciosos, mucho más condescendientes con el sueño del vecindario; pero era patente que no estaba al alcance de todos los bolsillos, en el momento de su lanzamiento o comercialización.

Cuando a mediados, más bien finales para algunos, de los ochenta la microinformática comienza a llegar a nuestras vidas, el sentimiento favorable y acogedor prima entre los investigadores sociales ocupados en calcular porcentajes verticales, horizontales y totales. Incluso si el uso del ordenador personal era estrictamente para mecanografiar con un procesador de textos los papeles manuscritos, la receptividad era casi unánime. Sin embargo, en fecha tan tardía como la de 1998 se sigue publicando la pregunta: ¿por qué usar ordenadores en la investigación cualitativa? No es otro el título que dan a su capítulo 3 Fielding y Lee (1998) y en él afrontan la «evaluación de las ventajas de

${ }^{16}$ Está por hacerse la historia de la práctica de la investigación social en su relación con las distintas fases tecnológicas. Una aportación reciente en esta línea la representa el estudio «Recording Technologies and the Interview in Sociology, 1920-2000» de Raymond M. Lee (2002), leccion inaugural del curso 2002-03 pronunciada en Royal Holloway, University of London, Surrey. 
CAQDAS $\gg{ }^{17}$. Bajo este epígrafe se encuentra el enfoque conceptual más elaborado de estos autores, su visión o perspectiva procesual. No hay ni ventajas ni inconvenientes abstractos, desligados del tiempo, de la circunstancia histórica y biográfica del investigador. $\mathrm{Ni}$ el software deja de evolucionar, ni el usuario es el mismo al comienzo que cuando se familiariza. Hay una tipología de usuarios por definir ${ }^{18}$ y de software. Unos y otros evolucionan.

De ahí la importancia de conceptos como el destacado aquí, por nosotros, de transición tecnológica; o los utilizados en la literatura referida: generación del investigador o generación del programa (Fielding y Lee 1998; Fielding 2000; Gibbs Friese y Mangabeira 2002). En el proceso de exposición a los recursos tecnológicos disponibles, a lo largo de la vida del investigador, la percepción del saldo resultante tras la inversión necesaria en tiempo de aprendizaje y gasto monetario varía. Esta anticipación lleva a algunos investigadores, cuyo horizonte laboral es más limitado, a descartar la opción del reciclaje. La insistencia en el condicionante de la experiencia vivida y la por vivir (horizonte vital) está presente en el análisis de Fielding y Lee (1998: 59) sobre las ventajas y los inconvenientes de los nuevos desarrollos tecnológicos para la investigación social cualitativa. Así dirán, por ejemplo, que: «también es sensato diferenciar lo que podríamos llamar ventajas e inconvenientes "anticipados" de los "validados experiencialmente"». O bien, la reiteración de su enfoque en esta otra cita de la misma fuente, que destacamos:

«Una perspectiva procesual se acomoda al carácter dinámico del ambiente de la investigación y de la negociación del lugar del CAQDAS dentro de éste. Esto es particularmente importante cuando examinamos las críticas que surgen de la experiencia previa. En numerosos casos han emergido soluciones técnicas a problemas experimentados por usuarios que participaron en nuestros grupos focalizados y que no estaban disponibles en el momento de nuestro trabajo de campo. Los propios usuarios también se hacen más sofisticados».

Cabe deducir de este punto de vista, que cualquier esfuerzo de inventario en este terreno estaría condenado de antemano a una caducidad tan rápida como el avance de las versiones de los productos tecnológicos y el ritmo del aprendizaje por parte de los usuarios. Aunque así fuera, nuestro punto de vista es que la reflexión metodológica sobre esta cuestión cumple una función necesaria: de documentación, también de un cierto control de posibles efectos no queridos, además de promover un proceso de transición en el que se tiene en cuenta una experiencia histórica heredada o una realidad social siempre compuesta de varias

17 CAQDAS responde a la expresión Computer Assisted Qualitative Data Analysis Software; i.e. software para el análisis de datos cualitativos asistido por ordenador.

18 Tanto en los escritos previos de Lee y Fielding $(1991,1995)$, como en los posteriores (Fielding y Lee 2002), se echa en falta una mayor definición tipologica de los usuarios de software cualitativo. No obstante, en la ponencia presentada por Ray Lee en el Primer Seminario de Investigación Avanzada Cualitativas Asistida por Ordenador, celebrado en Granada (22-23 Noviembre, 2001) organizado por CentrA, se esbozaba la distinción de tres tipos: naive adopters, old hands y craft users. 
generaciones. Nuestro propósito aquí va más allá de la actualización del listado de ventajas e inconvenientes ${ }^{19}$. Orientamos la indagación hacia el análisis de las posturas discursivas existentes entre los que se muestran favorables, contrarios o indecisos; sean estos usuarios, con mayor o menor experiencia, metodólogos, tecnólogos o tipos mixtos. Con ello, pretendemos dar contexto a la presentación de la postura defendida por Glaser, que se hace en el siguiente apartado.

Lo que sigue es un intento de elaboración tipológica centrado en los casos y en los procesos. Se parte de la combinación de los modos manuales y los modos asistidos de análisis en la investigación cualitativa. Adviértase que nuestra noción de análisis, entiende éste de manera omnipresente, en todos los momentos de la investigación (Valles 1997: 325-326). Cada vez más, la asistencia del ordenador y otras tecnologías aparece en las fases de preparación de proyectos, durante la realización del campo y en las tareas de análisis intenso final e informe. La tipología sintetiza una concurrencia de tradiciones y nuevos estilos de investigación en proceso. Reúne modos muy añejos y artesanales junto a prácticas con menos historia. Las generaciones de investigadores sociales que coexisten en la actualidad se ubican en alguno de los nichos tipológicos resultantes, aunque pueden haber ocupado ya otros o lo harán en el futuro. El casillero no ha de entenderse de manera estática, ni rígida. De hecho se cruzan en su elaboración las dimensiones pasada y presente de la experiencia investigadora de cada caso-tipo. Entendemos que puedan darse situaciones intersticiales, no representadas gráficamente en este primer cuadro. Se ha optado, como paso previo, por la tipología más sencilla, entre otras muchas trazadas. Lo que se pierde en detalle, debido a la reducción dicotómica de otros gradientes más amplios, se gana en claridad. Estos son los tipos y procesos que creemos más elementales (Cuadro 1).

\section{Cuadro 1}

TIPología I: Tipos básicos de analistas y procesos de transición tecnológica, según sus experiencias de investigación cualitativa en el pasado y en el presente

\begin{tabular}{|c|l|c|c|}
\hline \multicolumn{2}{|c|}{$\begin{array}{c}\text { PRACTICA } \\
\text { INVESTIGADORA }\end{array}$} & \multicolumn{2}{c|}{ EN EL PRESENTE } \\
\cline { 2 - 4 } PREDOMINANTE & $\begin{array}{c}\text { Modos } \\
\text { manuales }\end{array}$ & $\begin{array}{c}\text { Modos } \\
\text { tecnoasistidos }\end{array}$ \\
\hline \multirow{3}{*}{ EN EL PASADO } & $\begin{array}{l}\text { Modos } \\
\text { tecnoasistidos }\end{array}$ & $\begin{array}{c}\text { Tecnoarrepentidos } \\
\text { TRANSICIÓN INVERS }\end{array}$ & $\begin{array}{c}\text { Tecnovanguardistas } \\
\text { POSTRANSICIÓN }\end{array}$ \\
\cline { 2 - 4 } & $\begin{array}{l}\text { Modos } \\
\text { manuales }\end{array}$ & $\begin{array}{c}\text { Tecnófobos } \\
\text { PRETRANSICIÓN }\end{array}$ & $\begin{array}{c}\text { Tecnoentusiastas } \\
\text { TECNOTRANSICIÓN }\end{array}$ \\
\hline
\end{tabular}

${ }^{19}$ Consúltese sobre ello Fielding y Lee (1998) para el caso británico o Valles (2001) para el contexto sociológico español. 
Releyendo lo escrito años atrás sobre «los sistemas informáticos y el análisis cualitativo» (Valles 1997: 393-400), reparo en una serie de advertencias y recomendaciones tomadas del seminal trabajo de Tesch (1990). Una de ellas sirve para enfatizar lo que se quiere transmitir con el Cuadro 1. Esto es, «no descuidar el análisis intensivo centrado en el caso y en el proceso, por el exceso de atención en el análisis centrado en las variables o en las relaciones entre categorías conceptuales". Los casos-tipo destacados en este primer casillero tipológico son cuatro, que cabe leer así: a) el par tecnófobos-tecnoentusiastas, que comparten un pasado común en su biografía intelectual, la formación y el ejercicio profesional de manera más artesanal, aunque pueda haber variantes generacionales; y b) el par tecnovanguardistas-tecnoarrepentidos, cuyo hilo umbilical se halla también en un pasado de experiencias investigadoras asistidas por los medios tecnológicos disponibles.

La lectura complementaria que se sugiere presta más atención a los procesos de transición tecnológica, pero ayuda también a perfilar mejor los tipos mencionados. La diagonal principal marca los dos extremos del cambio tecnológico analizado: el estadio inicial de pre-transición y el final de pos-transición ${ }^{20}$. La diagonal secundaria recoge los dos procesos netos de transición, de cambio en curso o ya experimentado. El más importante, desde el punto de vista de la dirección del cambio social, la modernización en el contexto social más amplio, es la tecnotransición. Apócope de la expresión transición tecnológica empleado en aras del lenguaje deseablemente sucinto de los cuadros y representaciones esquemáticas. El primer plano o portavocía que se da a los llamados tecnoentusiastas, en este nicho tipológico, supone en algunos casos más deseo que realidad seguramente. Puede incluso que el entusiasmo tenga sus oscilaciones al alza y a la baja, según la experiencia de tecnoasistencia que se viva o se recuerde. En todo caso, en términos de proceso, en esta celdilla se representa en conjunto el camino real, transitado por unos como éxodo y por otros como complemento, sin cortar amarras con el pasado. En el extremo opuesto de la diagonal se alude a un proceso de transición inversa, contracorriente de la pauta descrita. Sus protagonistas, los tecnoarrepentidos o tecnodesengañados, han probado más las hieles que las mieles de las nuevas tecnologías hasta el punto de retornar en el presente a los modos manuales, después de una experiencia pasada de un cierto predomino de los modos tecnoasistidos. Sin duda, el arrepentimiento o la frustración no son exclusivos de ellos. Ya se ha advertido, respecto al tipo anterior, que el entusiasmo tiene sus altos y bajos en un proceso largo de aprendizaje, de formación continua e interminable cabría decir incluso.

${ }^{20}$ Conviene aclarar esta expresión «postransición». Ciertamente el pasado tecnoasistido, como práctica investigadora predominante posible en lo cualitativo, ha sido más difícil en épocas anteriores. Es necesaria una cierta adaptación de la literalidad del casillero. Se entiende mejor referido a los últimos años, para el caso de las generaciones más jovenes. Pero «tecnovanguardistas» ha habido en todas las épocas, por definición siempre una minoría. Van marcando el norte, al que llegan los primeros, sin apenas transición como estadio alargado que es para una mayoría siempre a la zaga. 
A pesar de los matices, anotados con el fin de aumentar la validez de esta propuesta tipológica, hay que reconocer sus limitaciones debidas a la reducción operada en su construcción, ya señaladas al comienzo de esta exposición. Por ello, se acompaña esta primera tipología con una segunda en la que ensayamos una definición complementaria. Adviértase cómo el concepto de transición tecnológica sigue siendo la categoría conceptual central. Pero no se descuida el análisis intensivo de los casos y los procesos, de una manera integrada. Hay una reducción en la perspectiva temporal, el foco se sitúa ahora sólo en el presente, pero se compensa con la referencia a los procesos y las variantes generacionales dentro del nuevo casillero (Cuadro 2).

El Cuadro 2 gana en detalle, pero pierde en claridad. Su lectura y comprensión lleva más tiempo. El autor arriesga más. Vale la pena, no obstante, por una ganancia añadida con provecho para el metodólogo: cualquier análisis ad-

Cuadro 2

TiPología II: Procesos de transición tecnológica y casos-tipo, según experiencias de investigación cualitativa en el presente

\begin{tabular}{|c|c|c|c|c|}
\hline & \multicolumn{3}{|c|}{ PRACTICAN MODOS MANUALES } \\
\hline & & МИСНо & POCO & NADA \\
\hline \multirow{6}{*}{$\begin{array}{l}\text { PRACTICAN } \\
\text { MODOS } \\
\text { TECNOASISTIDOS }\end{array}$} & \multirow{2}{*}{ МUСHO } & \multicolumn{3}{|c|}{$\begin{array}{l}\text { Procesos avanzados de tecnotransición o } \\
\text { post-transición y posturas de tecnofilia }\end{array}$} \\
\hline & & $\begin{array}{l}\text { (5) } \\
\text { Tecnoentusiastas } \\
\text { tradicionalistas }\end{array}$ & $\begin{array}{l}(6) \\
\text { Tecnoentusiastas } \\
\text { rupturistas }\end{array}$ & $\begin{array}{c}\text { (7) } \\
\text { Tecno- } \\
\text { vanguardistas }\end{array}$ \\
\hline & \multirow[b]{2}{*}{ POCO } & \multicolumn{3}{|c|}{$\begin{array}{l}\text { Procesos iniciales de tecnotransición, con variantes } \\
\text { generacionales de tecnoentusiasmo moderado }\end{array}$} \\
\hline & & $\begin{array}{c}(4) \\
\text { Artesanos } \\
\text { tecnoaperturistas } \\
\text { tímidos }\end{array}$ & $\begin{array}{l}\text { (3) } \\
\text { Tecnoartesanos } \\
\text { tibios }\end{array}$ & $\begin{array}{c}(2) \\
\text { Tecno- } \\
\text { principiantes }\end{array}$ \\
\hline & \multirow{2}{*}{$N A D A$} & \multicolumn{3}{|c|}{$\begin{array}{c}\text { Procesos pre-transicionales } o \text { de transición inversa } \\
\text { y posturas tecnófobas, tecnoescépticas } \\
\text { o de tecnodesengaño }\end{array}$} \\
\hline & & \multicolumn{2}{|c|}{$\begin{array}{l}\text { (1) } \\
\text { Artesanos tecnofobos } \\
\text { o tecnoescépticos }\end{array}$} & $\begin{array}{c}(0) \\
\text { Teóricos, } \\
\text { contramétodo }\end{array}$ \\
\hline
\end{tabular}


mite ulteriores elaboraciones; cualquier enfoque o interpretación se complementa con otros posibles. Este segundo intento de elaboración tipológica tampoco resulta definitivo. Ahora bien, puede someterse a prueba su grado de validez. Por ejemplo, comprobando si en él encuentran asiento las posturas defendidas por los distintos autores que han participado en el debate sobre los riesgos o las ventajas del uso de la tecnología en la investigación cualitativa. $\mathrm{O}$, también, ubicando en dicho cuadro los resultados obtenidos por Fielding y Lee $(1998,2002)$ acerca de las experiencias de los usuarios en el Reino Unido.

Empezaré explicando las limitaciones de este último test, y su descarte como prueba de fuego, a pesar de habernos servido de referencia para la confección de nuestras tipologías. En el primer estudio de los metodólogos citados, los usuarios de CAQDAS que participaron en los grupos focalizados fueron de cuatro tipos y en la siguiente proporción: «poco menos de la mitad (...) eran investigadores contratados, una cuarta parte eran profesores universitarios y el otro cuarto posgraduados, estando empleados los pocos individuos restantes en tareas de apoyo informático o papeles de asesoramiento» (1998: 2). Resumiendo, mitad escasa de investigadores profesionales no académicos, mitad académicos (unos profesores y otros jóvenes titulados), más unos pocos consultores. Reconocen no tener información precisa sobre el tiempo de uso de los programas, por parte de los participantes. No obstante, señalan que algunos son novicios, otros veteranos y algunos incluso evaluadores de programas. Pero el flanco más débil de su muestra, que los propios investigadores advierten, es que los usuarios «eran en su mayor parte early adopters del software»; y que tanto «los programas como la facilidad de su manejo por los usuarios se han desarrollado apreciablemente en el ínterin» (1998: 3). En el segundo estudio se informa, sobre todo, del aumento de los usuarios no académicos y procedentes de disciplinas distintas a las de las ciencias sociales. Pero tampoco se aporta información suficiente sobre los procesos y los casos de transición tecnológica o sobre la gama de posturas contempladas en el Cuadro 2 por nosotros.

A pesar de estas limitaciones, los trabajos de Fielding y Lee resultan extraordinariamente provechosos. Su atención preferente por las experiencias de los usuarios novicios, les lleva a ellos como metodólogos a adoptar una postura de relativa equidistancia ${ }^{21}$ respecto de los tecnovanguardistas o tecnoentusiastas más interesados (fabricantes de los programas, intermediarios de su comercialización y enseñanza: tecnólogos, mayoristas, consultores) y respecto de los tecnófobos o tecnoescépticos que ven peligrar su artesanía o su posición en el mercado del conocimiento y el reconocimiento ${ }^{22}$. Para ilustrar el contenido argumentativo de estas posturas polares, bastaría pulsar el discurso de uno de los

21 Consúltense, por ejemplo, las conclusiones del capítulo 3 de Fielding y Lee (1998). Una de ellas viene a decir que los usuarios consultados en su estudio rebajan tanto las loas como las críticas hechas a CAQDAS. Pero dejan claro que «los usuarios experimentaron dificultades y frustración en el aprendizaje y en el uso de los programas» (Fielding y Lee 1998: 84).

${ }^{22}$ Como dejó escrito Bourdieu (2003: 65-66) «el capital científico es un tipo especial de capital simbólico, capital basado en el conocimiento y el reconocimiento». 
extremos. Tomemos el caso de los más favorables a la fusión de la tecnología con la metodología. Reservamos el apartado siguiente para exponer las razones de Glaser, en tanto representante de los más reacios a la recepción de las nuevas tecnologías en la investigación cualitativa, pero particularmente en la metodología de la que es progenitor.

Chris Thorn es Director del Departamento de Servicios Técnicos, en el Centro Wisconsin para la Investigación Educativa de la Universidad de Wisconsin-Madison. Firma tres páginas de presentación en el número especial de la recién lanzada Qualitative Research Journal, en 2003. Este número especial recoge los materiales de la primera conferencia sobre Teaching Qualitative Methods, celebrada en Abril de 2003 en el centro universitario mencionado. Adviértase que se trata de una conferencia organizada por una novísima revista australiana, controlada por los Richards y de ahí el carácter monográfico de la conferencia, dedicada exclusivamente al software de la firma QSR. La justificación de esta restricción aparece también al comienzo del editorial escrito por Singh. A saber: «la discusión (...) se restringió al Software QSR - N6 y NVivopara evitar la focalización en las características del software», y hacerlo en «las cuestiones de investigación y enseñanza». Pretexto o no, en el Seminario ya referido de Granada, aquí en España en 2001, quizá se desvió un poco el debate hacia el software, pero fueron muy interesantes las críticas a los fabricantes. Entre ellas la falta de transferibilidad del trabajo analítico entre los programas.

Thorn ofrece, además, razones del rechazo al software por parte de los «instructores de métodos de nivel universitario» ${ }^{23}$; y una crítica a la concepción medieval del aprendizaje que lleva a pensar en Glaser, pero en general en las posturas y las experiencias investigadoras más tradicionales. Por ejemplo, se refiere al esfuerzo de los partidarios de la incorporación del software, en la enseñanza de algo que ha sido tradicionalmente «el dominio de un enfoque de métodos manuales al trabajo de investigación». Sin duda éste es el proceso en el que se está, también en España.

Otro ejemplo revelador de la postura discursiva que representa este autor (y de su opuesta) está en el uso de la palabra craft y en la, reiterada, referencia crítica a las formas tradicionales de enseñanza, que se hace patente en el extracto siguiente:

«Quizás mi uso de la palabra craft $^{24}$ pueda explicar parte de la reticencia a usar herramientas informáticas cualitativas que he visto y oído en discusiones con colegas. Parece haber una tradición de estudiantes que aprenden las

${ }^{23}$ La cita de Thom (2003: 7), observador participante natural, es harto elocuente: «Ha sido mi experiencia (y lo he discutido con quienes usan y desarrollan software analítico) que los instructores de métodos cualitativos de nivel universitario son a menudo indiferentes (o incluso hostiles) al software de análisis cualitativo. Se ve como demasiado complicado y "entorpeciendo el análisis". He oído quejas de que los programas "conducen a un cierre prematuro" o que "distancian en exceso de los datos" - a pesar del hecho de haber tenido poca o ninguna experiencia con el software en cuestión quien así se expresa».

${ }^{24}$ Craft se refiere, en este contexto, a oficio artesanal, manual. En el campo de la metodología sociológica resuena el célebre apéndice de C. Wright Mills «Sobre artesanía intelectual», «On Intellectual Craftmanship». 
habilidades y técnicas básicas del análisis cualitativo en la rodilla de un mentor. Hay también un sentimiento claro de que uno debiera aprender investigando. Se espera de lo estudiantes que vayan al campo con escasa formación práctica y encuentren la relación entre epistemología, método y técnica. Este enfoque de la formación y el aprendizaje en métodos cualitativos ha producido algo similar a los gremios de siglos anteriores. Tenemos cuadros de eruditos formados en procesos particulares y usando hand-crafted tools para replicar la forma de trabajar de sus mentores» (Thorn 2003: 7).

En el número especial de la revista citada aparecen otras aportaciones, cuya reseña en el editorial tiene un valor añadido para el analista de las líneas editoriales o posturas discursivas. Allí se glosa la que pudiera ser la postura mayoritaria de los participantes en la conferencia. Antes se alude a la contraposición de dos perspectivas «conflictivas y desarticuladas» sobre el ordenador, como epítome de lo tecnológico. La contraposición se expresa en términos de miedo y mística. Miedo a que la máquina ridiculice al investigador; o mecanice el proceso de análisis, a expensas de la reflexión e interpretación. $O$, por el contrario, «la creencia mística de que el ordenador producirá el análisis». Ante esta bifurcación (que recuerda algunos puntos del mapa trazado aquí de posturas tecnófobas y tecnófilas), se plantea una tercera vía, una posición intermedia: ni de terror tecnológico, ni de reverencia mística. Los recursos tecnológicos actuales —se acaba diciendo- sirven para pensar, abren posibilidades que antes no existían; pero también están abiertos a un uso inadecuado de los mismos, aunque éste sea vistoso o incluso creativo.

No es éste el único derrotero, hacia el espacio más neutro o equidistante de la metodología más contemporizadora, que se hace público desde las posiciones más favorables a la profusión de los recursos informáticos en la enseñanza de la metodología cualitativa. Queda anotado en este espacio constreñido de la comunicación vía artículo de revista. Un último apunte. La lectura de este documento, divulgado generosamente en el ciberespacio, hace que repare en un desafío no destacado en la síntesis de ventajas y desafíos elaborada en $2001^{25}$. Se trata del desafio docente que supone la integración de los nuevos recursos tecnológicos en la docencia de los métodos y técnicas cualitativos. La experiencia que se relata en esta fuente (de dificultades por las constricciones de tiempo y medios) coincide bastante con la explorada en el caso español ${ }^{26}$.

\section{LA POSTURA DE GLASER COMO EPICENTRO DEL DEBATE}

Lo escrito hasta aquí sirve para contextualizar el caso-tipo que la figura de Barney G. Glaser pueda representar en el panorama de procesos y casos so-

${ }^{25} \mathrm{El}$ documento electrónico referido puede consultarse en http://www.latrobe.edu.au/aqr/; para la síntesis de ventajas desafíos véase Valles (2001).

${ }^{26}$ El relato de las dificultades se acompaña de algunos ejemplos de cómo avanzar en la integración de la tecnología y la metodología de la investigación social cualitativa, entre los que destacan las contribuciones de Jackson (2003) y Di Gregorio (2003). 
ciológicos de transición tecnológica en el terreno de la investigación social cualitativa. La presentación de su postura tiene, así, una resonancia que creemos facilita la comprensión de la misma. En el resumen, se han avanzado ya algunas razones que avalan la pertinencia de un enfoque centrado en este autor, al que metafóricamente le ubicamos en el lugar más rompedor de una actividad dialéctica por analogía a la actividad sísmica, el epicentro. No se vea en ello el resultado de un trabajo bibliométrico convencional, sino más bien el fruto de una operación muestral de carácter estratégico, ilustrada, y apoyada incluso en la experiencia vivida. Daré un botón autobiográfico de esto último, como primer paso.

Esta es la historia, abreviada, de un primer gran temblor. En el Seminario sobre Investigación Avanzada Cualitativa Asistida por Ordenador, organizado por CentrA (Fundación Centro de Estudios Andaluces) en 2001, estaba previsto que asistiese Glaser. El terrorismo internacional del 11 de septiembre de ese año intervino en la modificación de esta previsión, primero hacia el formato videoconferencia y finalmente reduciéndola a una comunicación telefónica. El encuentro había reunido a usuarios cualificados de CAQDAS, desarrolladores de programas concretos y metodólogos. El broche final del seminario se esperaba fuese la mesa redonda con todos los conferenciantes que habían intervenido en las dos jornadas (22-23 noviembre), en la que Glaser disertaría sobre la grounded theory y los programas informáticos para la investigación cualitativa. La intervención de Glaser provocó una gran polémica y motivó sobre todo la réplica, en defensa de sus programas, de los creadores que estaban presentes. Por mi parte, aunque no tenía un programa que defender, sí tuve la sensación de jarro de agua fría que caía en un ambiente más tecnófilo que tecnófobo. La segunda pregunta que formulé a Glaser resume el sentido más general de su postura al respecto: «Sólo he anotado aspectos negativos del uso de ordenadores si el investigador quiere generar teoría, ¿hay algún aspecto positivo, alguna ventaja?». La respuesta fue concisa: «Como procesadores de texto sólo, pues inhiben la grounded theory. No son necesarios para ésta».

Destaco algunas de las anotaciones tomadas entonces. Una, «los ordenadores vuelven a poner lo cualitativo en lo cuantitativo». Dos, «los ordenadores ignoran la saturación de conceptos y sus propiedades». Tres, "pueden ser útiles en el análisis de datos cualitativos, donde la investigación va preconcebida, pero no en la grounded theory (GT) por su incompatibilidad e ineficacia». Cuatro, «son un malgasto de recursos y esfuerzos del investigador, la GT funciona más rápido». Cinco, «la grabación de las notas de campo es una pérdida de tiempo». Seis, «una parte vital de la GT es detenerse en cualquier momento y pasar a la ideación de los memos».

Estos brevísimos fragmentos del discurso de Glaser conviene completarlos y ponerlos en el contexto de su obra escrita. De hecho la invitación a la lectura de su último libro entonces (Glaser 2001) y a algunos otros anteriores (Glaser $1978 ; 1992 ; 1998)$ tuvo lugar desde el comienzo de la intervención. No faltaron tampoco las referencias de algunos de los otros conferenciantes a la obra primi- 
genia firmada con Anselm Strauss ${ }^{27}$ en 1967. La lectura o relectura de esta dilatada obra metodológica ayuda, sin duda, a comprender mejor las razones de las partes enfrentadas en esta polémica. Como ya se ha visto en el apartado anterior, el análisis de una postura acaba desvelando la contraria. Este es el caso, nuevamente. La irrupción de los programas informáticos para la investigación cualitativa ha llevado a Glaser a remachar, aún más, las lindes del que considera el territorio de la «grounded theory genuina ortodoxa» (Glaser 2001: 3). En ese empeño, la contraposición de la perspectiva de la GT con la más general del QDA (qualitative data analysis) resulta primordial para entender la postura reacia de este autor, fundamentada metodológicamente, a la asistencia tecnológica.

En su libro marrón ${ }^{28}$, el viejo profesor advierte de la variedad de perspectivas en lo cualitativo y del «positivismo» acechante. Aboga desde el principio por diferenciar su enfoque. La diferencia más destacada, respecto al conjunto de los otros métodos de análisis cualitativo, se resume en la contraposición de la conceptualización y la descripción: «GT existe en un nivel conceptual y está compuesta de hipótesis integradas mientras que los métodos QDA producen descripción con o sin descripción conceptual» (Glaser 2001: 1-2) ${ }^{29}$. La variedad de métodos de análisis cualitativo, respecto a los que diferencia su perspectiva, son los «positivistas, social constructivistas e interpretativistas». Contra todos ellos se dirige de manera especialmente contundente, en la obra citada, con el fin de parar la «cooptación y corrupción» (o la «confusión y fusión») de la GT por parte de los investigadores QDA ${ }^{30}$. Sin embargo, a mi juicio, hay algunas briznas de flexibilidad ( ¿o son momentos episódicos de flaqueza?) en su ortodoxia. Por ejemplo cuando afirma que la aminoración de la distinción puede ser beneficiosa en ocasiones. Se refiere a una suerte de trueque: autonomía (para la GT) a cambio de la tolerancia de las numerosas modalidades QDA que toman prestadas algunas nociones de GT (Glaser 2001: 4). No es moneda corriente este tono, en-

${ }^{27}$ No hay espacio aquí para exponer la polémica entre Glaser y Strauss, que puede leerse en la versión monográfica de Glaser (1992) y en los ecos más abreviados de autores menos implicados. Fielding y Lee $(1998: 35,38)$, por ejemplo, emplean la expresión Straussian Grounded Theory para sintetizar dicha polémica. Consideran el libro de 1992 una crítica «fundamentada y sólida» del trabajo de Strauss y Corbin (1990). Y concluyen subrayando que a pesar de lo significativo de la controversia «representa una disputa sobre énfasis y componentes particulares del que es ahora un abordaje muy desarrollado del análisis de datos cualitativos». Los escritos más recientes de Glaser no se habían publicado cuando va a imprenta el libro de Fielding y Lee.

${ }_{28}$ Entre los conocedores de la obra de Glaser, pasada y actual, se suelen utilizar las expresiones libro amarillo (yellow book) y libro marrón (brown book) para referirse a los textos impresos en 1978 y en 2001, respectivamente. La razón no es otra que el color de las tapas de sus encuadernaciones.

${ }^{29}$ Llega a hablar de «distinción poderosa», clave para superar las «confusiones crónicas entre teoría como un conjunto de conceptos integrados» (lo que equipara a la GT), y la «descripción conceptual» o las meras «descripciones» (que hace equivaler a QDA). Niveles que conviene distinguir, pues «cada cual tiene sus propiedades diferentes». Y adelanta que «muchos de los problemas y debilidades de la descripción no incumben/no procede a la concetualización» (Glaser 2001: 3).

${ }^{30}$ Hay un proceso de «adopción, adaptación, cooptación y corrupción» de la GT en las modalidades QDA, según Glaser. 
tre negociador y tolerante, en los escritos de este autor, donde son constantes los intentos de clarificar la tan traída y llevada distinción. Este es un botón de muestra con resonancia mertoniana ${ }^{31}$ :

«La consecuencia no anticipada de este descubrimiento de la teoría enraizada es un sobreénfasis en el enraizamiento que conduce muy fácilmente a la descripción QDA. Así el investigador, para permanecer enraizado, trata de describir los datos con precisión para producir evidencia y dar voz a los participantes. Este sobreénfasis distrae a muchos investigadores de la tarea de conceptualización ${ }^{32}$ en su celo por describir con precisión. Como resultado mientras la GT se aplica, muchos investigadores harán QDA que meramente describe pero lo llamarán GT» (Glaser 2001: 5).

La singularidad de su propuesta metodológica apunta, una y otra vez, a la generación de conceptos y su trabazón mediante hipótesis; al polo de la abstracción con raíces o conexiones empíricas frente al polo de la descripción que se obsesiona por el número de casos y el verbatim de los participantes e informantes. El enfoque, moderadamente alternativo, que plantea Glaser se fundamenta en su carácter trascendente: «GT trasciende el tiempo, el lugar y la gente de cualquiera y de todas las unidades maestrales y genera conceptualmente las pautas fundamentales que aportan hipótesis que pueden explicar el comportamiento de los participantes» (Glaser 2001: 5).

\section{MÁS ALLÁ DEL MERO CUESTIONAMIENTO DEL SOFTWARE}

En la contraposición, hecha por Glaser, de la GT con las otras perspectivas de análisis cualitativo se hallan ya algunas de las razones de la postura reacia de este autor al uso de los programas. Pero conviene hacer éstas más explícitas. Para ello, nada mejor que el capítulo 12 de su monografía de 1998, dedicado a actualizar lo escrito sobre anotaciones teoréticas (theoretical memos) veinte años antes. En la obra de 1998 puede apreciarse que el cuestionamiento del software se concreta en los programas tipo NUD*IST ${ }^{33}$. En este año no se había lanzado aún NVivo, que saldría en marzo de 1999 con una serie de mejoras en el aspec-

31 Dicha resonancia no debe hacer olvidar la disparidad de criterio en lo sustantivo, entre Merton y Glaser. Algo ya presente en la obra primera de Glaser y Strauss (1967), pero también en la más reciente de Glaser $(1998,2001)$ donde se vuelve a criticar a los "teóricos capitalistas», por su implicación en un modo de investigar donde prima la «teoría conjeturada forzada, preconcebida».

${ }^{32}$ La tarea de la conceptualización es lo que verdaderamente distingue a la GT del QDA, según este autor. Lo cual no se acaba de entender, cabalmente, sin la distinción entre códigos substantivos y códigos teoréticos expuesta por este metodólogo tiempo atrás (Glaser 1978: 55-82).

${ }^{33}$ Glaser (1998: 105) deja claro que también probo, en los años 60, las tarjetas MacBee y las de indexar. Acerca de éstos y otros recursos mecánicos de codificación ya había señalado, años antes, su adecuación para el modelo aritmético concepto-indicador pero no para el modelo comparativo constante concepto-indicador (Glaser 1978: 71-72). 
to del memoing. Estos detalles son importantes porque ayudan a comprender la postura de Glaser y la contraria, contrastando una y otra sobre un mismo software y en una misma época. Una razón metodológica principal que arguye Glaser (1998: 178) está en las restricciones «formales y sistemáticas» impuestas por el software a la anotación «libre, flexible y fluida» de ideas (el memoing ${ }^{34}$ ). Afirma haber hablado con los desarrolladores de NUD*IST, de quienes subraya su desconocimiento cabal de la grounded theory. La argumentación suena a que debiera ser alguien con experiencia en GT quien diseñe software específico, como así lo acaba escribiendo (Glaser 1998: 185-86).

Claro que esta condición también la pueden blandir los tecnólogos: exigir un conocimiento cabal del software para juzgarlo. Una aproximación a este juicio, por parte de peritos en la tecnología y la metodología cualitativas, se tiene en la valoración que hace Silvana di Gregorio, metodóloga antes que consultora de software, con experiencia de investigación cualitativa en la época preinformática. Su testimonio sobre lo mejorable del programa NVivo en relación a la GT, tiene el valor añadido del contexto en el que pronunció su valoración (Di Gregorio, 2003). Baste señalar aquí que buena parte de los aspectos a mejorar se refieren al memoing. Aunque son muchas las posibilidades de anotación (memoing) en NVivo - se viene a decir-, esta característica «está algo "oculta" en comparación con las opciones de codificación» ${ }^{35}$. Comparto este juicio, más apreciable si se compara con Atlas.ti por ejemplo. Ahora se comprende un poco mejor la suspicacia de Glaser hacia el modelo anterior a NVivo, las versiones de NU*DIST disponibles antes de 1998 (más rudimentarias aún en el aspecto del memoing). Con todo, mi valoración más sopesada es que aun habiendo todavía margen para la mejora de las nuevas versiones el obstáculo mayor a superar se halla en la formación técnica, de métodos, del analista. La importancia del memoing en la GT, y en el análisis cualitativo en general, es un elemento clave que algunos usuarios de los nuevos programas parecen olvidar o desconocer. Sobre todo los que evitan en lo posible tener que leer el material a analizar y su preocupación mayor es la de obtener un fichero de información manejable con los programas de análisis cuantitativo.

Ahora bien, Glaser no sólo se muestra contrario al software tipo NU*DIST. Hay referencias explícitas a una categoría más amplia, «las tecnologías», donde sobresale el cuestionamiento de la grabación magnetofónica (taping) y la trascripción (typing) ${ }^{36}$.

${ }^{34}$ El trabajo alrededor de la toma de notas y su codificación es clave en la perspectiva de la grounded theory. Glaser $(1967 ; 1978)$ ya había desvelado su tecnología preinformática (de fichas de papel + papel carbón + tijeras...). Y sigue prefiriéndola a las opciones informáticas de los años 60 y las actuales. De estas últimas, a juzgar por sus escritos, conoce el programa NU*DIST pero no se sabe muy bien con qué grado de experticia.

${ }^{35}$ Un modo de comprobar esto es tratar de escribir un informe, un artículo con NVivo. Si no trabajas al mismo tiempo con un esquema de la escritura, te pierdes en la maraña de códigos y documentos (memos o no), tan fáciles de crear.

${ }^{36}$ Merece leerse a fondo el capítulo 7 Taping, en el que se defiende contracorriente la opción de no grabar las entrevistas. Hay todo un despliegue de reflexión metodológica para argumentar esta 
«Las tecnologías, especialmente los ordenadores, que producen inadvertidamente su propio modelo de producto ideacional debido a sus constricciones, son una fácil excusa para el abandono del poder completo del $\mathrm{me}$ moing (...)

Pero estoy abierto a la computarización si se me demuestra cómo hacer la comparación constante, la delimitación a partir de la intercambiabilidad de índices, la saturación, otras delimitaciones, la relevancia pautada latente, la culminación teorética (...) el muestreo (...); cómo parar la total cobertura descriptiva, cómo mantener el nivel conceptual, cómo insertar y ordenar anotaciones memo (...) sin perder el tiempo (...)

La constante aligeración y delimitación del proceso de la grounded theory en su camino a la teoría, se pierde por las recogidas copiosas y el proceso de los ordenadores y las grabadoras. Así la grabación y la computerización ofrecen la red de seguridad de la tecnología, arrojando un producto formulado en lugar de un análisis más libre de grounded theory»(Glaser 1998: 185-186).

En lo que respecta a las tecnologías de audiograbación, podrá objetarse que la postura de Glaser no hace justicia al papel desempeñado por éstas en la mejora de las técnicas de investigación, desde los tiempos de Carl Rogers hasta la actualidad. La parte de razón que le asiste a nuestro autor estaría, por un lado, en que no pretende hablar del único estilo o modo de análisis cualitativo. La expresión "grounded theory package» avisa de la concepción enteriza que se da a esta perspectiva singular, que precisa de mayores dosis de «flexibilidad y de libertad» que el común de la investigación cualitativa. Hay, no obstante, una pretensión de liberar al investigador de técnicas de producción mecánica que están dentro de «la Bailía o jurisdicción de la verificación de teoría». En otras palabras, se trata de la conocida pretensión de incrementar lo emergente frente a lo preconcebido.

\section{A MODO DE CONCLUSIÓN: EL CONTRAPUNTO DE GLASER A UNA RELACIÓN POLÉMICA}

La postura de Glaser, ultraortodoxa quizá para algunos, cabe entenderla positivamente cumpliendo una función muy conveniente. Esto es, representaría un cierto ideal o modo ejemplar de entender la perspectiva de la grounded theory. Al mismo tiempo, los esfuerzos de simplificación, divulgación o innovación que vemos en escritos como los de Strauss y Corbin $(1990,1997)$ o en los más recientes de Gibbs (2002) o Di Gregorio (2003) sirven para alumbrar una gama de aproximaciones, que se suman a las líneas de investigación abiertas por otros

postura, que ya despuntaba en Glaser (1992) respecto a la trascripción. Mi posición sobre esto último puede verse en Valles (2002: 138). Glaser (1998: 111) remarca que «la riqueza de la grounded theory proviene de los datos off-the-record». La grabación, «ralentiza la codificación, el análisis y el muestreo teorético, por tanto la relevancia». Y reitera que el objetivo en la GT no es la cobertura descriptiva, sino la concepttual. 
tantos autores (Fielding y Lee 1998, 2002; Jackson 2003). Los seguidores puristas o practicantes eclécticos de la grounded theory -en tanto herederos del legado intelectual concebido por Glaser, sobre todo, y por Strauss- pueden parecerse más a un estilo que a otro o incluso a ninguno.

Resulta comprensible el desasosiego que rezuma la obra de Glaser. Hay, ciertamente, un riesgo claro de pérdida de la pureza de la propuesta primigenia. Pero ello no debiera valorarse de manera negativa. Después de todo, el alumbramiento de la grounded theory por sus progenitores en 1967 (aunque su gestación venía de atrás ${ }^{37}$ ), lo hizo posible el mestizaje de las dos tradiciones o escuelas: la de Chicago y la de Columbia. Más aún, el «descubrimiento» se presentaba como un «tercer approach» que combinaba una cierta dosis de la codificación sistemática previa del análisis de contenido tradicional, con la práctica más abierta de la inspección del material a analizar sin dicha codificación (Valles 1997: 346).

Concluyo anotando que las palabras habladas y escritas del admirado profesor Glaser están cargadas de razón, de experiencia metodológica vivida. Su virtuosismo metodológico, al igual que el del profesor Strauss en vida, es un modelo o prototipo que ha servido y seguirá sirviendo de referencia en la investigación social. Por ello ha ejercido una gran atracción sobre los creadores y desarrolladores de programas informáticos para el análisis de material cualitativo. Por cierto, programas muy rudimentarios en sus primeras versiones, mejorados poco a poco y ad infinitum, con los costes de actualización subsiguientes (en tiempo de aprendizaje y gasto continuo). Hay, por tanto, parte de razón y parte de exageración o defensa a ultranza por parte de unos y otros. El análisis asistido por ordenador tiene ventajas, pero también desafíos, siendo el docente uno principal; y no sólo cuando el material es de naturaleza cualitativa.

${ }^{37}$ Resulta obligado referirse al menos a la principal investigación social que antecede a la obra netamente metodológica de 1967, cual es Awareness of dying, publicada en 1965 y avanzada en 1964. Pero conviene no olvidar las raíces intelectuales, psicosociológicas, de Glaser y Strauss. Basta fijarse en las referencias bibliográficas de sus escritos. 


\section{REFERENCIAS BIBLIOGRÁFICAS}

BARRY, C. A. (1998): «Choosing qualitative data analysis software: Atlas/ti and Nudist Compared», Sociological Research Online, vol. 3, n. 2 , http://www.socresonline.org.uk/ socresonline/3/3/4.html .

Bourdieu, P. (2003): El oficio de científico. Ciencia de la ciencia y reflexividad. Barcelon, Anagrama.

Cabrera, J. (1987): «Una aplicación informática para el estudio de casos», Revista Española de Investigaciones Sociológicas, 40, pp. 161-176.

CRESWELL, J. W. (1998): Qualitative inquiry and research design. Choosing among five traditions, London, Sage.

COFFEY, A.; HolbrooK, B. and AtKInson, P. (1996): «Qualitative Data Analysis: Technologies and Representations», Sociological Research Online, vol. 1, n. ${ }^{\circ} 1$, http://www.socresonline.org.uk/socresonline/1/1/4.html .

Di GREGORIO, Silvana (2003): «Teaching grounded theory with QSR NVivo», Qualitative Research Journal, Special issue 2003, pp. 79-95.

FIELDING, N. G. \& LEE, R. M. (eds.) (1991): Using Computers in qualitative research. London, Sage.

- (1998): Computer analysis and qualitative research. London, Sage. New technologies for social research Series.

- (2002): «New patterns in the adoption and use of qualitative software», Field Methods, vol. 14, n. ${ }^{\circ}$, pp. 197-216.

GiBBs, G. R. (2002): Qualitative data analysis. Explorations with NVivo. Buckingham, Open University Press.

GibBs, G. R.; Friese, S. \& Mangabeira, W. C. (2002): «The use of new technology in qualitative research. Introduction to issue 3 (2) May of $F Q S$ ». Forum Qualitative Sozialforschung / Forum: Qualitative Social Research). http://www.qualitative-research.net/fqs/fqs-eng.htm .

GLASER, B. G. (1965): «The constant comparative method of qualitative analysis», Social Problems, 12, pp. 436-445.

- (1978): Theoretical sensitivity: advances in the methodology of grounded theory, Mill Valley, CA: Sociology Press.

- (1992): Basics of grounded theory analysis: emergence vs. forcing, Mill Valley, CA: Sociology Press.

- (1993) (ed.) Examples of grounded theory: a reader, Mill Valley, CA: Sociology Press.

- (1998): Doing grounded theory: issues and discussions, Mill Valley, CA: Sociology Press.

- (2001): The grounded theory perspective: conceptualization contrasted with description, Mill Valley, CA: Sociology Press.

- (2002): «Conceptualization: On theory and theorizing using grounded theory», International Journal of Qualitative Methods, 1 (2). Article 3. Disponible en: http://www.ualberta.ca/ ijqm/spanish/spframeset.html .

Glaser, B. G. \& Strauss, A. L. (1964): «Awareness contexts and social interaction», American Sociological Review, vol. 29, n. 5 (oct.), pp. 669-679.

- (1965): Awareness of Dying. Chicago, Aldine.

- (1967): The Discovery of Grounded Theory. Chicago, Aldine.

HAMILTON, D. (1994): «Traditions, preferences, and postures in applied qualitative rese- 
arch», en N. K. Denzin \& Y. Lincoln (eds.) Handbook of qualitative research. Thousand Oaks, California: Sage, pp. 60-69.

JACKSON, K. (2003): «Blending technology and methodology: a shift toward creative instruction of qualitative methods with NVivo", Qualitative Research Journal, Special issue 2003, pp. 96-110.

KelLe, U. (1997): «Theory Building in Qualitative Research and Computer Programs for the Management of Textual Data», Sociological Research Online, vol. $2, \mathrm{n}^{\circ}{ }^{2}$, http://www.socresonline.org.uk/socresonline/2/2/1.html .

LEE, R. M. \& FIELDING, N. G. (1991): «Computing for qualitative research: options, problems and potential», en N. G. Fielding y R. M. Lee (eds.) (1991) Using Computers in qualitative research. London, Sage, pp. 1-13.

- (1995): «User's experiences of qualitative data analysis software», en U. Kelle (ed.) Computer-aided qualitative data analysis. London, Sage, pp. 29-40.

LONKILA, M. (1995): «Grounded theory as an emerging paradigm for computer-assisted qualitative data analysis", en U. Kelle (ed.) Computer-aided qualitative data analysis. London, Sage, pp. 41-51.

MochmanN, E. (1985): «Análisis de contenido mediante ordenador aplicado a las ciencias sociales», Revista Internacional de Sociología, 431, pp. 11-43.

OTTENBERG, S. (1990): «Thirty years of fieldnotes: changing relationships to the tex», en R. Sanjeck (ed.) Fieldnotes. The makings of anthropology. New York, Ithaca: Cornell University Press, pp. 139-160.

STRAUSS, A. \& CORBIN, J. (1990): Basics of qualitative research: Grounded theory procedures and techniques. London, Sage.

- (1994): «Grounded theory methodology: an overview», en N. K. Denzin \& Y. Lincoln Handbook of Qualitative Research, Thousand Oaks, California: Sage, pp. 273-285.

- (1997): Grounded theory in practice. London, Sage.

TESCH, R. (1990): Qualitative research: analysis types and software tools. New York, The Falmer Press.

THORN, Ch. (2003): «Why a conference?», Qualitative Research Journal, Special issue 2003, pp. 6-8.

VALLES, M. S. (1997): Técnicas cualitativas de investigación social. Reflexión metodológica y práctica profesional. Madrid, Síntesis (reimpreso en 1999, 2000 y 2003).

- (2000): «La grounded theory y el análisis cualitativo asistido por ordenador», en García Ferrando, M.; Ibáñez, J. y Alvira, F. El análisis de la realidad social. Métodos y técnicas de investigación, Madrid, Alianza, pp. 575-604.

- (2001): «Ventajas y desafíos del uso de programas informáticos (e.g. ATLAS.ti y MAXqda) en el análisis cualitativo. Una reflexión metodológica desde la grounded theory y el contexto de la investigación social española». Ponencia presentada en el Primer Seminario sobre Investigación Avanzada Cualitativa Asistida por Ordenador, organizado por CentrA en Granada: 22-23 Noviembre, editada en Documentos de Trabajo Serie Sociología (S2001/05) de la Fundación Centro de Estudios Andaluces, 2002, 26 págs.

WeItzman, E. A. \& Miles, M. B. (1995): Computer programs for qualitative data analysis. London, Sage. 


\section{RESUMEN}

La obra metodológica de Glaser $(1978,1992,1998,2001,2002)$ constituye una postura contracorriente de la atracción de las nuevas tecnologías, experimentada por algunos investigadores sociales en los últimos veinte años. Resulta, además, paradójica pues la tradición metodológica de la grounded theory (Glaser y Strauss, 1967) se ha invocado como fundamento metodológico por los tecnólogos en la presentación del software diseñado para asistir en el análisis cualitativo. Baste mencionar los programas ATLAS.ti, NVivo o MAXqda.

Por otro lado, la biografía intelectual de Glaser ofrece algunas claves de la superación de la polémica entre las perspectivas cuantitativistas y cualitativistas. Autor crítico con sus maestros de Columbia, se ha venido mostrando polémico después y en su madurez actual, también con la corriente principal de los investigadores cualitativistas anglosajones. Con ellos mantiene una pugna, tratando de reivindicar la perspectiva original y más ortodoxa de la GT (grounded theory) frente a los intentos de subsumirla en el magma del enfoque QDA (qualitative data analysis) de la investigación cualitativa. Por nuestra parte, tratamos de ahondar en dicha polémica, explorando con detenimiento el punto de vista de Glaser, cuya postura consideramos epicentro de un campo abierto de investigación y reflexión metodológica de gran actualidad y relevancia.

\section{PALABRAS Clave}

Metodología cuantitativa, tecnología, teoría fundamentada.

\section{ABSTRACT}

Glaser's methodological writings $(1978,1992,1998,2001,2002)$ constitute a countercurrent attitude on the attraction towards new technologies experienced by some social researchers in the past twenty years. Moreover, this attitude could be seen as paradoxical because the methodological tradition of Grounded Theory (Glaser and Strauss, 1967) has been invoked as a methodological foundation by the developers of software designed to assist qualitative analysis. It will be sufficient to mention the programmes ATLAS.ti, NVivo or MAXqda.

On the other hand, Glaser's intellectual biography offers some clues in order to overcome the discussion between qualitative and quantitative perspectives. $\mathrm{He}$ was critical with his teachers of Columbia. Later and now, in his present maturity, he has also been critical with the mainstream Anglo-Saxon qualitative researchers. He maintains a wrestling with them, setting the more orthodox and original perspective of the GT (grounded theory) against the intents of subsuming it in the magma of the QDA approach (qualitative data analysis) to qualitative investigation. Our work tries to make a deep assessment of this contro- 
versy, exploring Glaser's point of view carefully. So, we think Glaser's attitude is in the middle of a very relevant open field of investigation and methodological reflections.

\section{KEY WORDS}

Qualitative methodology, technology, grounded theory. 